\title{
Inhibition of skin squamous cell carcinoma proliferation and promote apoptosis by dual silencing of NET-1 and survivin
}

\author{
ZHOU-JING JI ${ }^{1 *}$,JIAN-LI WANG ${ }^{1 *}$ and LI CHEN $^{2}$ \\ ${ }^{1}$ Department of Dermatology and Venereology, Affiliated Hospital of Nantong University; \\ ${ }^{2}$ Department of Pathological Anatomy, Nantong University, Nantong, Jiangsu, P.R. China
}

Received April 3, 2015; Accepted May 29, 2015

DOI: $10.3892 /$ or.2015.4062

\begin{abstract}
The simultaneous silencing of multiple upregulated genes is an attractive and viable strategy to treat many incurable diseases including cancer. In the present study, skin squamous cell carcinoma (SSCC) tissue microarray was constructed and the expression of NET-1 and survivin was identified. The high expression of NET-1 and survivin gene in SSCC was confirmed as an important event for the formation and development of the cancer. A total of 100 primary SSCC patients were included in the present study. Expression of NET-1 and survivin in cancer cells was evaluated immunohistochemically in tissue microarrays. The interaction between NET-1 and survivin in SSCC by co-immunoprecipitation was subsequently verified by producing the siRNA sequence targeting the single gene (siRNA-NET-1 and siRNA-survivin) as well as NET-1 and survivin gene (one-chain-double-target siRNA). The levels of NET-1 and survivin mRNA and protein expression in A431 cells were detected by RT-qPCR and western blotting, and the expression of related genes including vascular endothelial growth factor (VEGF), cortactin, Bcl-2, caspase-3 and -8 was identified using RT-qPCR. The protein localization and expression of NET-1 and survivin in A431 cells were documented by immunohistochemistry and immunofluorescence staining. The proliferation and apoptosis of A431 cells were detected by CCK- 8 assay and flow cytometry (FCM). The tissue microarray showed that NET-1 and survivin were highly expressed in SSCC, while the correlation analysis showed NET-1 expression was positively associated with survivin. In addition, we reported that using the one-chain-double-target siRNA conjugate composed of NET-1 and survivin siRNA sequences in the same backbone inhibited proliferation and promoted apoptosis
\end{abstract}

Correspondence to: Professor Li Chen, Department of Pathological Anatomy, Nantong University, 19 Qixiu Road, Nantong, Jiangsu 226001, P.R. China

E-mail: bl1@ntu.edu.cn; chenli_bingli@yahoo.com

*Contributed equally

Key words: skin squamous cell carcinoma, A431 cells, NET-1, survivin, one-chain-double-target siRNA of SSCC. The one-chain-double-target siRNA showed further downregulation on NET-1 and survivin mRNA and protein levels compared with NET-1 siRNA or survivin siRNA. It also exhibited greater suppression on proliferation and triggering of apoptosis in A431 cells than NET-1 siRNA or survivin siRNA. This result may be explained by the significant downregulation of VEGF, cortactin and $\mathrm{Bcl}-2$, and upregulation of caspase-3 and -8. NET-1 and survivin were overexpressed in SSCC and an interaction between NET-1 and survivin was identified. The one-chain-double-target siRNA appears to be superior in inhibiting cell proliferation and promoting apoptosis compared with the single target siRNA. NET-1 and survivin may have correlative signaling pathways with VEGF, cortactin, Bcl-2, caspase-3 and -8. Simultaneous silencing of NET-1 and survivin using one-chain-double-target siRNA thus provides an advantageous alternative in the development of therapeutics for SSCC.

\section{Introduction}

Skin squamous cell carcinoma (SSCC) is a malignancy originating from epithelial keratinocyte, in which predisposing factors are associated with ultraviolet radiation, exposure to chemicals, the application of immunosuppressive drugs, trauma, scar and certain precancerous diseases. At present, the pathogenesis of the tumor is not entirely clear, but its ability for excessive proliferation and inhibition of apoptosis has been identified. As one of the most common skin malignant tumors, its incidence has been on the increase worldwide (1), and the age of patients is gradually becoming younger. Surgery is the most common and effective method of treatment for SSCC. However, radiotherapy, chemotherapy and scarring subsequent following surgery are of great concern to most patients (2). Thus, the study of its mechanism, prevention and treatment has become a hot topic in the field of dermatology.

Dysregulation of the balance between cell proliferation and apoptosis is a pro-tumorigenic principle in SSCC. The NET-1 gene is a new member of the molecules of the tetraspan superfamily (TM4SF), which is characterized by the existence of four predicted transmembrane domains that delimit two extracellular regions of unequal size (3). The gene is involved in signal transduction, cell adhesion, migration, proliferation and differentiation $(4,5)$. It was found to be overexpressed in certain tumors, such as SSCC (6), gastric cancer (7), 
cervical carcinoma (8), colorectal adenocarcinoma (9) and ovarian carcinoma (10). Previous findings have shown that the knockdown of NET-1 by RNA silencing and the antisense technique inhibit proliferation and infiltration of human skin squamous carcinoma cells. The NET-1 gene plays a role in the proliferation of SSCC and is associated with cancer cell motility, suggesting a function of the gene in the development of skin cancer (11).

In recent years, it has been found that the occurrence, development and recurrence of SSCC is closely associated with cell apoptosis disorder (12). Survivin, a member of the inhibitor of apoptosis protein (IAP) family, has been shown to inhibit apoptosis, enhance proliferation and promote angiogenesis. Due to its upregulation in malignancy and its key role in apoptosis, proliferation and angiogenesis, survivin is currently attracting considerable attention as a new target for anticancer therapies (13). In several animal model systems, the downregulation of survivin or inactivation of its function has been shown to inhibit tumor growth. Strategies under investigation to target survivin include antisense oligonucleotides, siRNA, ribozymes, immunotherapy and small molecular weight molecules. Survivin has been suggested as an attractive target for new anticancer interventions.

RNA interference (RNAi) is the phenomenon in which siRNA of 21-23 nt in length silences a target gene by binding to its complementary mRNA and triggering its degradation. The potent knockdown of specific gene sequences makes siRNA a promising therapeutic strategy $(14,15)$. Recent findings have shown that RNAi targeting NET-1, survivin, vascular endothelial growth factor (VEGF) and Bcl-2 transfected into A431 cells inhibited tumor cell proliferation, promoted apoptosis, and achieved a good tumor-suppressor effect in animal experiments.

Since the occurrence and development of cutaneous squamous cell carcinoma is a multistep complex process involving a variety of factors, there are some limitations for single oncogene inhibition. In the present study, the bulk cutaneous squamous cell carcinoma tissue chip was identified and the proliferation gene NET-1 or anti-apoptotic gene survivin was found to be important in the formation and development of cancer. Furthermore, a one-chain-double-target siRNA was constructed aiming at the NET-1 and survivin genes on the basis of screening effective single-target NET-1 siRNA and survivin siRNA sequences. The gene silencing effect of the one-chain-double-target siRNA in cutaneous squamous cell carcinoma and its effect on tumor proliferation and apoptosis were measured in in vitro studies. Additionally, the siRNA-NC group was established in the experiment to exclude the effect of the transfection reagent on the results, and a control group was established to demonstrate the effects of siRNA. The study aimed to investigate the mechanism of action of one-chain-double-target siRNA, expand RNAi technology, and provide a foundation for the development of one-chain-double-target siRNA technology. The results of the present study provided experimental evidence and technical support for the continuous innovation of the development of one-chain-multi-target siRNA nucleic acid drugs. Additionally, the results identified a new approach for the prevention and treatment of cutaneous squamous cell carcinoma.

\section{Materials and methods}

Patients and follow-up. This was a retrospective study based on archived materials. The study group comprised 100 SSCC patients who were diagnosed with SSCC and underwent curative resection in the Affiliated Hospital of Nantong University and the Nantong Cancer Hospital (Jiangsu, China) between January, 2007 and December, 2011. The patients were selected according to the following criteria: ii) primary SSCC; and ii) previously untreated with surgery as the first treatment. Therefore, analysis of the data in this series reflects the actual impact of the tumor biology on the clinical outcome.

The study was approved by the Ethics Committee of the Affiliated Hospital of Nantong University, and all the patients provided written informed consent.

Tissue microarray (TMA) construction. A representative section of the SSCC specimens used for creating tissue microarray were selected by two experienced pathologists, using hematoxylin (Invitrogen-Life Technologies, Carlsbad, CA, USA) and formalin-fixed and paraffin-embedded eosin-stained sections. Detailed clinical and pathological information of SSCC patients is provided in Table I. We used $1.0-\mathrm{mm}$ core tissue biopsies and took tissues from paraffin-embedded tissue blocks to new recipient blocks (contained 100 samples). The recipient blocks were cut and placed on slides.

Immunohistochemical (IHC) staining and scoring system. After deparaffinizing and rehydrating, the slides were treated with $3 \% \mathrm{H}_{2} \mathrm{O}_{2}$ solution for $15 \mathrm{~min}$ at room temperature to block endogenous peroxidase. The slides were then soaked in sodium citrate buffer $(10 \mathrm{mM}$ sodium citrate, $0.05 \%$ Tween- 20 , $\mathrm{pH}$ 6.0) at $96^{\circ} \mathrm{C}$ for $5 \mathrm{~min}$ for antigen retrieval. After blocking using BSA (Sigma, St. Louis, MO, USA), the following antibodies were used: mouse monoclonal antibody for NET-1 (1:100), mouse polyclonal antibody for survivin (1:100) (Santa Cruz Biotechnology, Inc., Santa Cruz, CA, USA). Antibodies were added to the slides, kept overnight at $4^{\circ} \mathrm{C}$ and then incubated at room temperature with biotinylated secondary antibody (Santa Cruz Biotechnology, Inc.) for $30 \mathrm{~min}$, and then HRP-streptavidin for $15 \mathrm{~min}$. After 3,3'-diaminobenzidine (DAB) (Sigma) staining, the results were graded for intensity (1, pale yellow; 2, yellow and 3, brown) and the percentage of positive cells [0 $(\leq 5 \%), 1(6-25 \%), 2(26-50 \%), 3(51-75 \%)$ and $4(>75 \%)]$ with discrepancies was resolved by consensus. The grades were multiplied to determine a score. The scores of tumors were defined as: $0(-), 1-3(+), 4-5(++),>5(+++)$.

Cell culture. A431 cells were grown in Dulbecco's modified Eagle's medium (DMEM; Invitrogen-Life Technologies) supplemented with $10 \%$ fetal bovine serum (FBS; Gibco-Life Technologies, Carlsbad, CA, USA), $2 \mathrm{mM}$ L-glutamine, $100 \mathrm{U} / \mathrm{ml}$ of penicillin and $100 \mu \mathrm{g} / \mathrm{ml}$ of streptomycin. The cell lines were purchased from the Institute of Cell Biology, Chinese Academy of Sciences. The cell cultures were maintained at $37^{\circ} \mathrm{C}$ in a humidified incubator (Fuyilian, China) with $5 \% \mathrm{CO}_{2}$.

siRNA, shRNA expression vector design, construct and shRNA expression vector stable transfection. On the basis 
Table I. The relationship between the expression of NET-1 and survivin and clinicopathological parameters in 100 cases of SSCC.

\begin{tabular}{|c|c|c|c|c|c|c|c|c|c|c|c|}
\hline \multirow[b]{2}{*}{ Characteristics } & \multirow[b]{2}{*}{ No. } & \multicolumn{4}{|c|}{ NET-1 } & \multirow[b]{2}{*}{ P-value ${ }^{a}$} & \multicolumn{4}{|c|}{ Survivin } & \multirow[b]{2}{*}{ P-value ${ }^{a}$} \\
\hline & & $(-)$ & $(+)$ & $(++)$ & $(+++)$ & & $(-)$ & $(+)$ & $(++)$ & $(+++)$ & \\
\hline \multicolumn{12}{|l|}{ Age (years) } \\
\hline$\leq 63$ & 45 & 2 & 7 & 16 & 20 & \multirow[t]{2}{*}{0.762} & 3 & 6 & 16 & 20 & \multirow[t]{2}{*}{0.811} \\
\hline$>63$ & 55 & 2 & 9 & 19 & 25 & & 2 & 8 & 21 & 24 & \\
\hline \multicolumn{12}{|l|}{ Diseased region } \\
\hline Head and face & 74 & 3 & 10 & 26 & 35 & \multirow[t]{4}{*}{0.619} & 2 & 8 & 31 & 33 & \multirow[t]{4}{*}{0.735} \\
\hline Body & 5 & 0 & 1 & 2 & 2 & & 1 & 1 & 1 & 2 & \\
\hline Arms and legs & 7 & 0 & 2 & 3 & 2 & & 1 & 1 & 2 & 3 & \\
\hline Externalia & 14 & 1 & 3 & 4 & 6 & & 1 & 4 & 3 & 6 & \\
\hline \multicolumn{12}{|c|}{ Tumor thickness (mm) } \\
\hline$\leq 5$ & 87 & 4 & 16 & 33 & 34 & \multirow[t]{2}{*}{0.004} & 5 & 14 & 36 & 32 & \multirow[t]{2}{*}{0.002} \\
\hline$>5$ & 13 & 0 & 0 & 2 & 11 & & 0 & 0 & 1 & 12 & \\
\hline \multicolumn{12}{|l|}{ TNM staging } \\
\hline I & 42 & 3 & 12 & 12 & 15 & \multirow[t]{4}{*}{0.021} & 4 & 11 & 16 & 11 & \multirow[t]{4}{*}{0.006} \\
\hline II & 51 & 1 & 4 & 22 & 24 & & 1 & 3 & 21 & 26 & \\
\hline III & 5 & 0 & 0 & 1 & 4 & & 0 & 0 & 0 & 5 & \\
\hline IV & 2 & 0 & 0 & 0 & 2 & & 0 & 0 & 0 & 2 & \\
\hline \multicolumn{12}{|c|}{ Pattern of organization } \\
\hline Classic SSCC & 55 & 3 & 6 & 18 & 28 & \multirow[t]{4}{*}{0.032} & 4 & 5 & 18 & 28 & \multirow[t]{4}{*}{0.039} \\
\hline Acantholysis & 20 & 0 & 3 & 11 & 6 & & 0 & 1 & 13 & 6 & \\
\hline Spindle cell & 14 & 0 & 0 & 3 & 11 & & 0 & 0 & 4 & 10 & \\
\hline Verrucous SSCC & 11 & 1 & 7 & 3 & 0 & & 1 & 8 & 2 & 0 & \\
\hline \multicolumn{12}{|c|}{ Lymph node metastasis } \\
\hline Present & 4 & 0 & 0 & 1 & 3 & \multirow[t]{2}{*}{0.047} & 0 & 0 & 0 & 4 & \multirow[t]{2}{*}{0.001} \\
\hline Absent & 96 & 4 & 16 & 34 & 42 & & 5 & 13 & 36 & 40 & \\
\hline
\end{tabular}

${ }^{\text {aPearson's }} \chi^{2}$ test, ${ }^{\mathrm{b}} \mathrm{p}<0.05$ for statistical significance. SSCC, skin squamous cell carcinoma; TNM, tumor-node-metastasis.

Table II. siRNA sequences.

\begin{tabular}{lll}
\hline Gene name & Strand & Sequence (5'-3') \\
\hline siRNA-NET-1 & Positive-sense & 5'-UGUGGUCUUUGCUCUUGGUUUCCdTdT-3' \\
siRNA-survivin & Antisense & 5'-ACACCAGAAACGAGAACCAAAGGdTdT-3' \\
& Positive-sense & 5'-UCUUUGUGACCCGGUUCAGdTdT-3' \\
siRNA-NET-1 and survivin & Antisense & 5'-AGAAACACUGGGCCAAGUCdTdT-3' \\
& Positive-sense & 5'-UGUGGUCUUUGCUCUUGGUUUCCUCUUU \\
& Antisense & GUGACCCGGUUCAGdTdT-3' \\
siRNA-NC & & 5'-ACACCAGAAACGAGAACCAAAGGdTdT-3' \\
& Positive-sense & 5'-AGAAACACUGGGCCAAGUCdTdT-3' \\
& Antisense & 5'-CTCACTAACCTCCAACCCCCTG-3' \\
\hline
\end{tabular}

of an optimization principle of siRNA, a sequence-specific siRNA targeting NET-1 (NET-1 siRNA) or survivin (survivin siRNA) was designed, and a dual gene targeting siRNA (one-chain-double-target siRNA) for NET-1 and survivin was assessed in vitro. Chemically produced oligonucleotides were obtained from Biomics Biotechnology Corp. (Nantong, China), and identified using PCR and DNA sequencing. Sequences of all the siRNAs and shRNA are shown in Table II. 
Table III. Oligonucleotide primers used for RT-qPCR analyses.

Gene

Primer sequence (strand)

Product size (bp)

\begin{tabular}{|c|c|c|}
\hline NET-1 & $\begin{array}{l}\text { 5'-GTGGCTTCACCAACTATACG-3'(+) } \\
\text { 5'-GACTGCATTAGTTCGGATGT-3'(-) }\end{array}$ & 191 \\
\hline Survivin & $\begin{array}{l}\text { 5'-ACCGCATCTCTACATTCAAG-3'(+) } \\
\text { 5'-CAAGTCTGGCTCGTTCTC-3'(-) }\end{array}$ & 113 \\
\hline VEGF & $\begin{array}{l}\text { 5'-GACATCTTCCAGGAGTACC-3'(+) } \\
\text { 5'-TGCTGTAGGAAGCTCATATCTC-3'(-) }\end{array}$ & 197 \\
\hline Cortactin & $\begin{array}{l}\text { 5'-AGCCGTCGCCCTGTACGACT-3'(+) } \\
\text { 5'-GTACCGGCCCTTGCACACCC-3'(-) }\end{array}$ & 127 \\
\hline Bcl-2 & $\begin{array}{l}\text { 5'-GGCTGGGATGCCTTTGTG-3'(+) } \\
\text { 5'-GCCAGGAGAAATCAAACAGAGG-3'(-) }\end{array}$ & 64 \\
\hline Caspase-3 & $\begin{array}{l}\text { 5'-AGAACTGGACTGTGGCATTGAG-3'(+) } \\
\text { 5'-GCTTGTCGGCATACTGTTTCAG-3'(-) }\end{array}$ & 191 \\
\hline Caspase-8 & $\begin{array}{l}\text { 5'-CGCAAAGGAAGCAAGAAC-3'(+) } \\
\text { 5'-TTGAGCCCTGCCTGGTGT-3'(-) }\end{array}$ & 361 \\
\hline GAPDH & $\begin{array}{l}\text { 5'-TGCACCACCAACTGCTTAGC-3'(+) } \\
\text { 5'-GGCATGGACTGTGGTCATGAG-3'(-) }\end{array}$ & 87 \\
\hline
\end{tabular}

VEGF, vascular endothelial growth factor; GAPDH, glyceraldehyde-3-phosphate dehydrogenase.

Briefly, A431 1.5×105 cells/ml (Bogoo, China) were seeded in multi-well plates (Corning Costar Corp., Cambridge, MA, USA) and were grown overnight until they reached $70-80 \%$ confluence. The abovementioned siRNA was transfected into A431 cells with Lipofectamine ${ }^{\mathrm{TM}} 2000$ reagent (Invitrogen-Life Technologies) according to the manufacturer's instructions. A431 cells were transfected with siRNA-survivin, siRNA-NET-1, siRNA-NET-1 and survivin or siRNA-NC, while the negative control was cultured using DMEM with $10 \%$ FBS.

Immunoprecipitation assay. A431 cells in the logarithmic growth phase were collected with trypsin and lysed in a pre-chilled RIPA buffer $\left(1 \mathrm{ml} / 10^{7}\right.$ cells; Beyotime, Jiangsu, China) for $1 \mathrm{~h}$ agitation at $4^{\circ} \mathrm{C}$. Protein $\mathrm{G}$ agarose beads (Sigma) were prepared by washing twice with phosphate-buffered saline (PBS) (KeyGen Biotech, Jiangsu, China) and restoring to a $50 \%$ slurry bead suspension with a RIPA buffer. Prior to immunoprecipitation, the cell lysate was pre-cleared by adding $50 \mu \mathrm{l}$ of bead slurry $/ \mathrm{ml}$, incubated at $4^{\circ} \mathrm{C}$, agitated for $10 \mathrm{~min}$ and centrifuged for $10 \mathrm{~min}$ at $10,000 \mathrm{xg}$ at $4^{\circ} \mathrm{C}$. The supernatant was transfered to a new tube. Mouse anti-human NET-1 antibody $(4 \mu \mathrm{g})$ was then added to $0.5 \mathrm{ml}$ pre-cleared cell lysate followed by incubation with agitation for $3 \mathrm{~h}$ on ice. Normal rabbit IgG and no IgG were used as controls. Fifty microliters of $50 \%$ slurry beads were added and rocked for $1 \mathrm{~h}$ at $4^{\circ} \mathrm{C}$. After the samples were centrifuged at $10,000 \mathrm{x}$ g for $15 \mathrm{sec}$, the beads were washed twice with $1 \mathrm{ml}$ RIPA buffer and then three times with $1 \mathrm{ml}$ PBS to remove detergents. The beads were then resuspended in a $60 \mu \mathrm{l}$ sample buffer, and boiled at $95^{\circ} \mathrm{C}$ for 5 min. Western blotting was performed with mouse anti-human survivin and mouse anti-human NET-1 antibodies to examine whether survivin was combined with beads and NET-1 was precipitated with survivin, respectively.

Reverse transcription-quantitative polymerase chain reaction $(R T-q P C R)$. Total RNA was isolated from A431 cells using TRIzol reagent (Invitrogen-Life Technologies), and then submitted to a $25 \mu \mathrm{l}$ PCR reaction in the presence of $12.5 \mu \mathrm{l}$ of 2X Master Mix, $1 \mu \mathrm{l}$ of each Primer Mix $(10 \mu \mathrm{M} / \mathrm{ml})$, $0.5 \mu 1$ of 50X SYBR-Green I and $4 \mu \mathrm{l}$ RNA according to the One-Step kit (Quantace, Australia). The PCR mixtures were first subjected to $30 \mathrm{~min}$ at $42^{\circ} \mathrm{C}$ for reverse transcription and initially denatured for $10 \mathrm{~min}$ at $95^{\circ} \mathrm{C}$ and then to 40 cycles of amplification with the following cycling parameters: $20 \mathrm{sec}$ at $95^{\circ} \mathrm{C}, 30 \mathrm{sec}$ at $55^{\circ} \mathrm{C}$, and $30 \mathrm{sec}$ at $72^{\circ} \mathrm{C}$. The primer pairs for each gene were designed with Primer Premier 5.0 software. Glyceraldehyde-3-phosphate dehydrogenase (GAPDH) served as an internal control for PCR. Primer sequences are provided in Table III.

Western blot analysis. After siRNA transfection for $48 \mathrm{~h}$, A431 cells in 6-well plates (Corning Costar Corp.) were lysed in RIPA buffer (Beyotime Institute of Biotechnology, China). The amount of total cell proteins was determined by BCA kit (Beyotime). Protein ( $25 \mu \mathrm{g}$ ) was separated by sodium dodecyl sulfate-polyacrylamide (SDS-page) separation gel and electroblotted onto PVDF membranes (Pharrnacai, Covington, KY, USA). For A431 proteins, the membrane was blocked with 5\% (wt/vol) milk and probed with a mouse-anti-NET-1 monoclonal antibody (1:500 dilution) and a mouse-anti-survivin polyclonal antibody (1:1,000 dilution), (Santa Cruz Biotechnology, Inc.) at 
$4^{\circ} \mathrm{C}$ overnight. The membrane was washed with Tris-buffered saline with Tween 20 (TBST; KeyGen Biotech), incubated with DyLight 800-labeled antibody to mouse $\operatorname{IgG}(1: 5,000)$ for $2 \mathrm{~h}$, and the membrane was scanned by the Bio-Rad imaging system for semi-quantitative analysis. An enhanced chemiluminescence (ECL) reagent was used to incubate the immune complex and signals were collected by ChemiDoc XRS (Bio-Rad, Hercules, CA, USA). Developed membranes were semi-quantitatively analyzed by scanning volume density using an ImageJ densitometer (National Institutes of Health). Results were expressed as optical volume density corrected by $\beta$-actin for loading. The size of target protein was measured by a comparison with protein molecular weight markers (Bio-Rad Laboratories, Ltd.).

Cell proliferation assay. Proliferation of the A431 cell line was measured using a CCK-8 detection kit (Dojindo, Japan). CCK-8 was applied at $10 \mu \mathrm{l} /$ well on the 1-3 days after transfection followed by $2 \mathrm{~h}$ incubation at $37^{\circ} \mathrm{C}$. The absorbance at $450 \mathrm{~nm}$ was determined by a microplate reader, model 680 (Bio-Rad). The samples were assessed in triplicate and differences among the controls and test groups were analyzed.

Annexin V-FITC apoptosis assay and flow cytometry. Briefly, after incubation for $48 \mathrm{~h}$ following transfection, the cells were collected and washed with PBS. The extent of apoptosis was measured by an Annexin V-FITC apoptosis detection kit (Beyotime Institute of Biotechnology) according to the manufacturer's instructions. The cells were washed with PBS twice, gently resuspended in an Annexin $\mathrm{V}$ binding buffer and incubated with Annexin V-FITC/PI in the dark for $15 \mathrm{~min}$ and analyzed by flow cytometry using CellQuest software (BD Biosciences, San Jose, CA, USA). The fraction of the cell population in different quadrants was analyzed using quadrant statistics.

Immunohistochemical staining. After incubation for $48 \mathrm{~h}$ following transfection, the A431 cells were incubated with primary antibodies [mouse monoclonal antibody for NET-1 (1:100) and mouse polyclonal antibody for survivin (1:100) (Santa Cruz Biotechnology, Inc.) for $30 \mathrm{~min} \mathrm{RT}$ ] at $4^{\circ} \mathrm{C}$ overnight followed by the standard avidin-biotin-peroxidase complex technique. Staining was visualized using a $\mathrm{DAB}^{+}$ substrate chromogen solution and hematoxylin QS counterstain. Images were captured as five fields of view.

Immunofluorescence assay. The expression of NET-1 and survivin was determined by immunofluorescence using specific antibodies (Cell Signaling Technology, Beverly, MA, USA). Briefly, A431 cells were grown into 60-80\% confluence on coverslips in 6-well plates. The cells were then fixed with $4 \%$ paraformaldehyde (KeyGen Biotech) for $30 \mathrm{~min}$. The fixed cells were permeabilized in 1\% BSA-supplemented PBS containing $0.5 \%$ Triton X-100 (Sigma) for $30 \mathrm{~min}$, washed and incubated overnight at $4^{\circ} \mathrm{C}$ with mouse-anti-NET-1 monoclonal antibody (1:200 dilution) and mouse-anti-survivin polyclonal antibody (1:200 dilution) (Santa Cruz Biotechnology, Inc.) as the primary antibodies. The cells were then washed extensively and incubated for $1 \mathrm{~h}$ at room temperature with secondary antibody. A goat anti-rabbit IgG-conjugated with FITC was used as the secondary antibody at a dilution of 1:100. The samples were counterstained with Hoechst 33258 and photographed using a confocal microscope (BX51; Olympus, Tokyo, Japan).

Statistical analysis. Experiments were performed independently at least three times. One-way ANOVA and t-test analyses were utilized to identify differences between groups. Statistical analysis was performed with SPSS software 16.0. $\mathrm{P}<0.05$ was considered to indicate a statistically significant result.

\section{Results}

\section{NET-1 and survivin expression in SSCC}

Preparation of tissue microarray. We successfully prepared the SSCC tissue microarray paraffin blocks, and each case was selected as a representative area of $H \& E$ sections. Each tissue microarray contained 100 sites. The tissue microarray results showed that the organization structure of the sites was well preserved, without any obvious necrosis organization. Additionally, the chips were arranged in an orderly manner (Fig. 1a and b).

NET-1 and survivin expression in SSCC and surgically removed breast cancer tissue. NET-1 and survivin were positively expressed in the cytoplasm and/or the cell membrane, stained as tan or brown (Fig. 1e and f). NET-1 and survivin were not expressed in surgically removed breast cancer tissue (Fig. 1c and d). The NET-1 positive rate was $96 \%$ in the SSCC group, but negative in the control group $(\mathrm{p}<0.001)$. The survivin positive rate was $95 \%$ in the SSCC group, but negative in the control group $(\mathrm{p}<0.001)$.

Relationship between the expression of NET-1 and survivin and clinicopathological characteristics in SSCC. The results showed that there were statistical differences between the expression intensity of NET-1 and survivin and tumor thickness, tumor-node-metastasis (TNM) stage, tissue types, and lymph node metastasis in SSCC $(\mathrm{p}<0.001)$. However, there were no significant associations between NET-1, survivin expression and other clinicopathological characteristics (p>0.05) (Table I).

Correlation between NET-1 and survivin. Due to the high expression of NET-1 and survivin in SSCC, we used Pearson's correlation analysis to assess the correlation between NET-1 and survivin protein expression. The percentage of positivity of NET-1 and survivin was detected and the result showed that there was positive correlation between the expression of NET-1 and survivin $\left(r^{2}=0.49, \mathrm{p}<0.05\right.$, Fig. 2$)$.

Association between NET-1 and survivin in A431 cells is identified using an immunoprecipitation assay. The relationship between NET-1 and survivin was investigated in A431 cells using an immunoprecipitation assay (Fig. 3). NET-1 protein was recognized by the NET-1 antibody in the anti-survivin group. The specificity was further supported by the fact that no corresponding protein bands were observed in any $\mathrm{IgG}$ group. The total protein was used as a positive control for NET-1 and survivin proteins. Survivin was recognized by the survivin antibody in the anti-NET-1 group, confirming a direct interaction between NET-1 and survivin in A431 cells. This also confirmed the correlation between the expression 


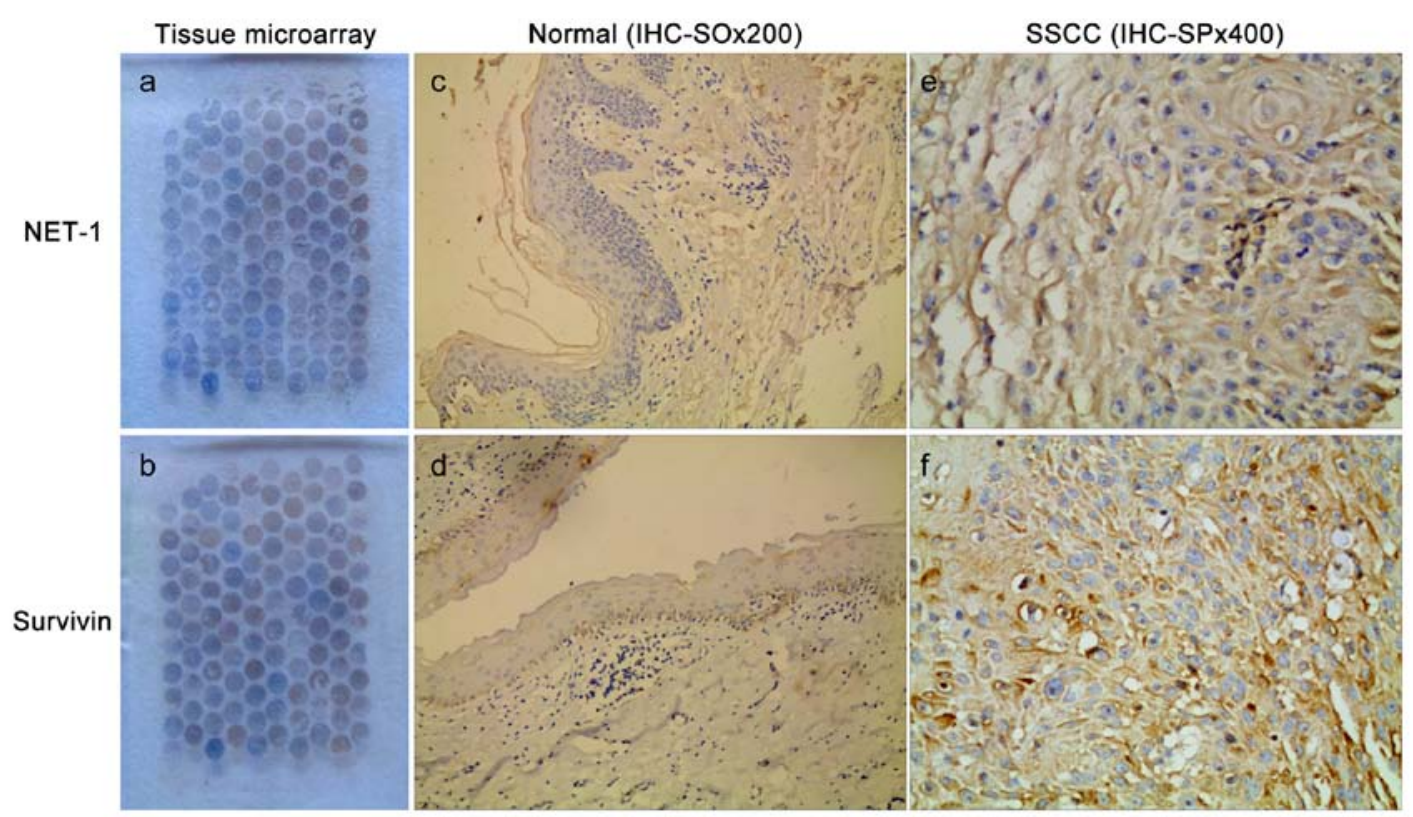

Figure 1. NET-1 and survivin expression in SSCC tissue microarray (a and b, tissue microarray; $c$ and d, IHC-SP, magnification, x200; e and f, IHC-SP, magnification, $\mathrm{x} 400$ ). SSCC, skin squamous cell carcinoma.

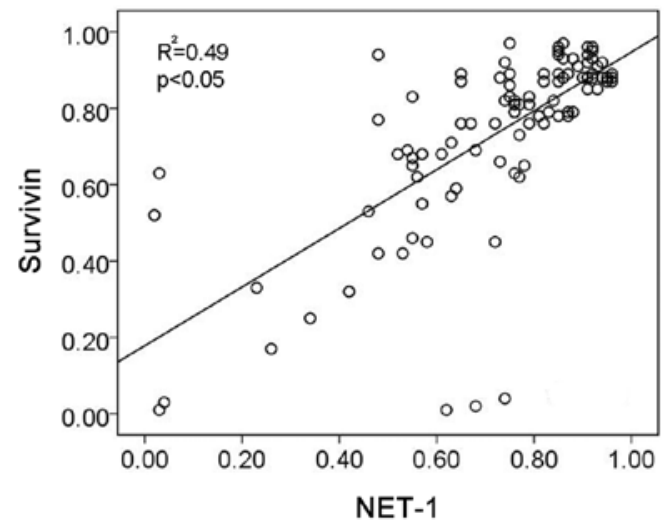

Figure 2. The correlation between NET-1 and survivin of SSCC. SSCC, skin squamous cell carcinoma.

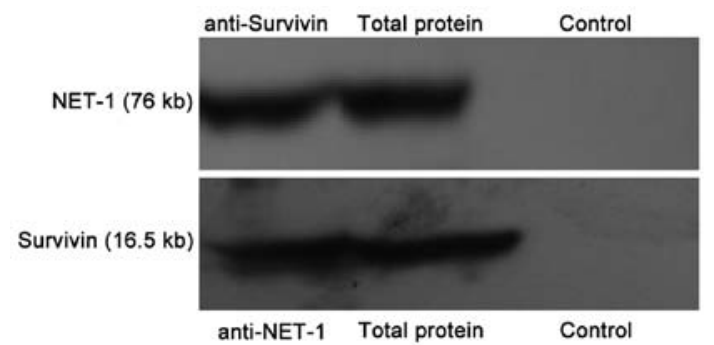

Figure 3. The NET-1 and survivin protein expression in the A431 cell co-immunoprecipitation product by western blotting.

of NET-1 and survivin at the SSCC organization level in the present study.

Effects of NET-1 SiRNA, survivin SiRNA and one-chaindouble-target siRNA on NET-1 and survivin expression in A431

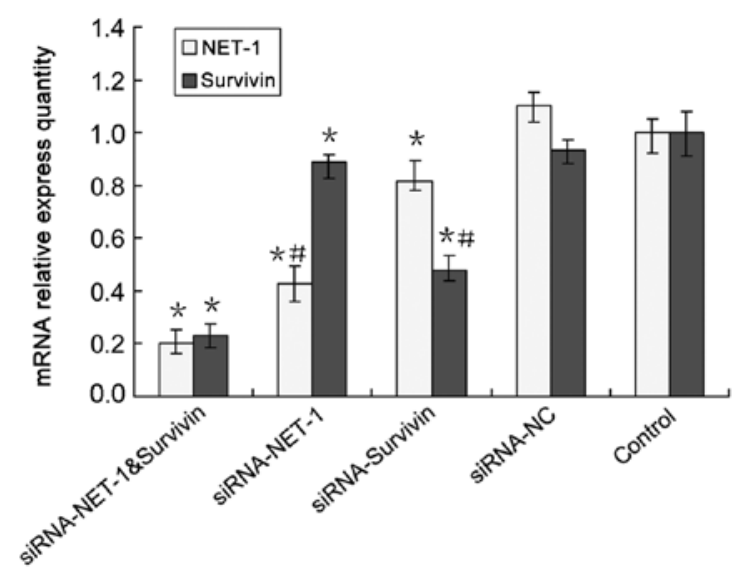

Figure 4. NET-1 and survivin expression in A431 cell transfected every siRNA at $48 \mathrm{~h}$ in mRNA levels ("p $<0.05$ vs. control; ${ }^{*} \mathrm{p}<0.05 \mathrm{vs}$. siRNA-NET-1\&survivin).

cells. After the A431 cells were transfected with siRNA for $48 \mathrm{~h}$, the levels of mRNA were determined by RT-qPCR while the protein expression of NET-1 and survivin was assessed using western blot analysis. When compared to the untreated group, NET-1 expression was decreased by $57 \%$ at the mRNA level for the cells transfected with NET-1 siRNA, and by $54 \%$ at the protein level (Figs. 4 and 5), indicating the effective silencing at the mRNA and protein levels. Similarly, survivin expression was effectively inhibited by survivin siRNA at the mRNA and protein levels by 52 and 58\% (Figs. 4 and 5), respectively. Notably, we found that the NET-1 siRNA significantly decreased survivin expression at the mRNA level by $11 \%$ (Fig. 4) and protein level by 27\% (Fig. 5). Similarly, survivin siRNA significantly decreased NET-1 expression at the mRNA level by $19 \%$ (Fig. 4) and the protein level by $15 \%$ (Fig. 5). This result showed for the first time that, 
A

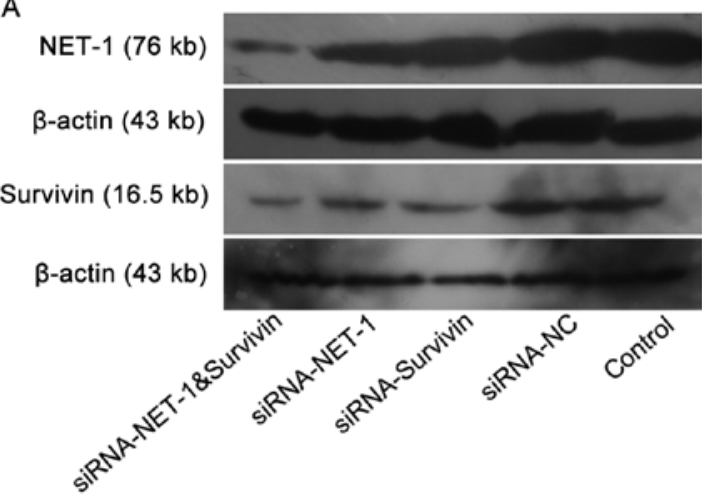

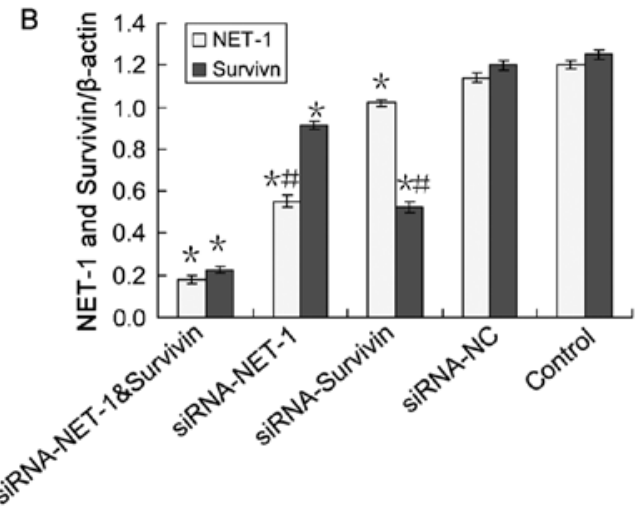

Figure 5. NET-1 and survivin expression in A431 cells transfected with siRNA at $48 \mathrm{~h}$ at the protein levels (A) The protein expression of NET-1 and survivin after transfection with western blotting. (B) Histogram ("p $<0.05$ vs. control; $" \mathrm{p}<0.05$ vs. siRNA-NET-1\&survivin).
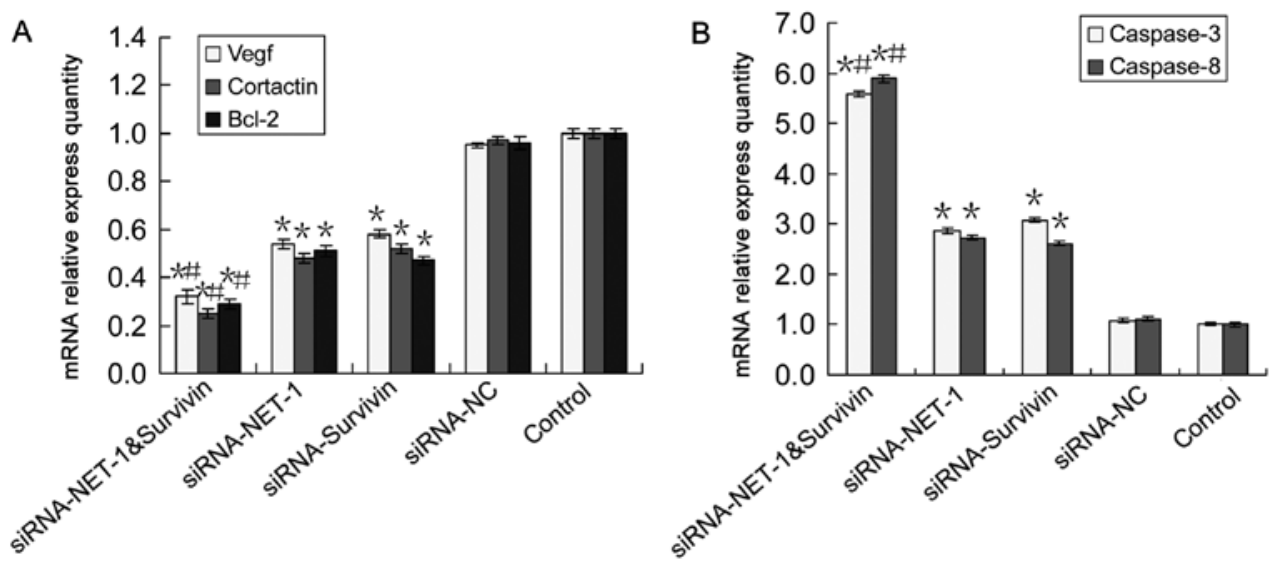

Figure 6. Vegf, cortactin, Bcl-2, caspase-3 and -8 expression in A431 cell transfected every siRNA at $48 \mathrm{~h}$ in mRNA levels. (A) Histogram of VEGF, cortactin and Bcl-2 expression. (B) Histogram of caspase- 3 and -8 expression ( $\mathrm{p}<0.05$ vs. control; ${ }^{*} \mathrm{p}<0.05$ vs. siRNA-NET-1\&survivin).

inhibiting NET-1 signaling reduced survivin expression and inhibiting survivin signaling reduced NET-1 expression.

Subsequently, we examined the effect of one-chaindouble-target siRNA on the expression of NET-1 and survivin, respectively. The results showed that NET-1 mRNA and protein levels were downregulated by $80 \%$ (Fig. 4) and $85 \%$ (Fig. 5), respectively, which was in concordance with that of the protein level of NET-1 siRNA. When compared to NET-1 siRNA or survivin siRNA alone, the one-chain-double-target siRNA showed a higher inhibition of survivin mRNA expression of up to $77 \%$ (Fig. 4) and of the protein level of up to $82 \%$ (Fig. 5), suggesting a marked effect of dual gene-targeted siRNA on survivin protein expression. No statistically significant difference was observed in the expression of the mRNA and protein siRNA-NC and blank control groups.

Effects of NET-1 siRNA, survivin siRNA and one-chain-doubletarget siRNA on VEGF, cortactin, Bcl-2, caspase-3 and -8 $m R N A$ expression in A431 cells. As shown in Fig. 6, NET-1 siRNA, survivin siRNA and one-chain-double-target siRNA inhibited the VEGF, cortactin and Bcl-2 expression at the mRNA level in comparison to the untreated group $(\mathrm{p}<0.05)$. By contrast, NET-1 siRNA, survivin siRNA and one-chain-double-target siRNA increased caspase-3 and - 8 expression at mRNA level in comparison to the untreated group ( $\mathrm{p}<0.05)$. In addition, compared to NET-1 siRNA or survivin siRNA alone, the one-chain-double-target siRNA was more effective in inhibiting and increasing the mRNA gene expression $(\mathrm{p}<0.05)$. There was no statistically significant difference in the expression of mRNA and protein between siRNA-NC and blank control groups ( $\mathrm{p}>0.05)$.

\section{Effect of siRNAs on A431 cell proliferation and apoptosis} Effect of NET-1 siRNA, survivin siRNA and one-chain-doubletarget siRNA on A431 cell proliferation. We examined the effect of silencing of NET-1 and survivin on cell proliferation of A431 cells. The absorbance values of the A431 cells at 24, 48 and $72 \mathrm{~h}$ after the transfection with NET-1 siRNA or survivin siRNA were significantly lower than those of the untreated cells (Fig. 7). There was no significant difference between the absorbance values of cells treated with NET-1 siRNA and that of survivin siRNA. The absorbance value of A431 cells treated with one-chain-double-target siRNA was significantly lower than the cells treated with NET-1 siRNA or survivin siRNA at 24,48 and $72 \mathrm{~h}$, respectively. There was no statistically significant difference in A431 cell proliferation between the siRNA-NC and blank control groups.

Effect of NET-1 siRNA, survivin siRNA and one-chaindouble-target siRNA on A431 cell apoptosis. Annexin V-FITC staining and flow cytometric analysis were performed to evaluate 
A

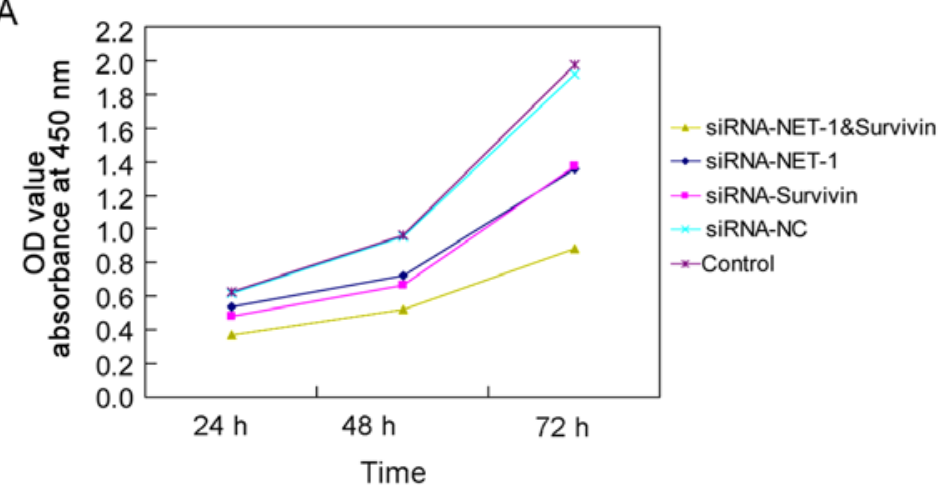

B

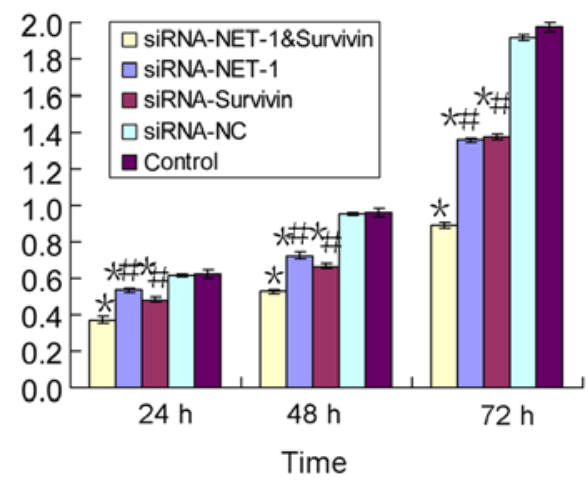

Figure 7. The growth level of A431 cells transfected with siRNA. (A) The growth curve of A431 cells transfected with siRNA by CCK-8. (B) Analysis results of A431 cell growth curve ( $\mathrm{p}<0.05$ vs. control; ${ }^{*} \mathrm{p}<0.05$ vs. siRNA-NET-1\&survivin).

A

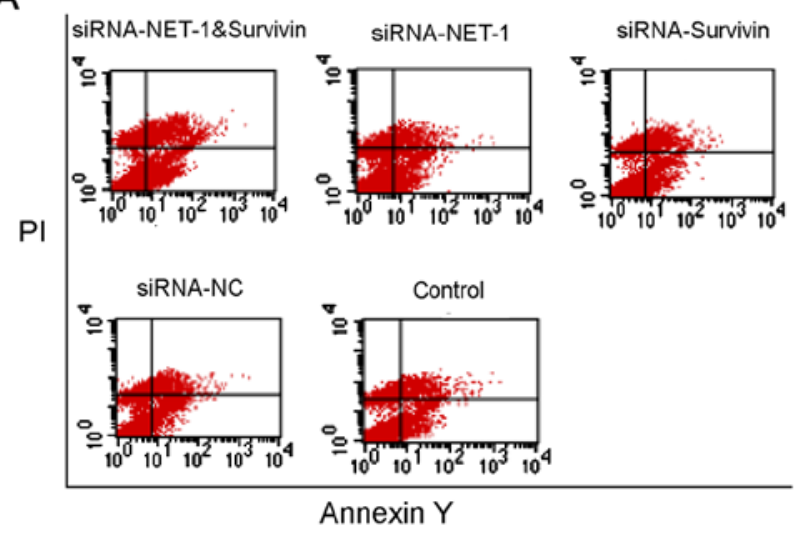

B

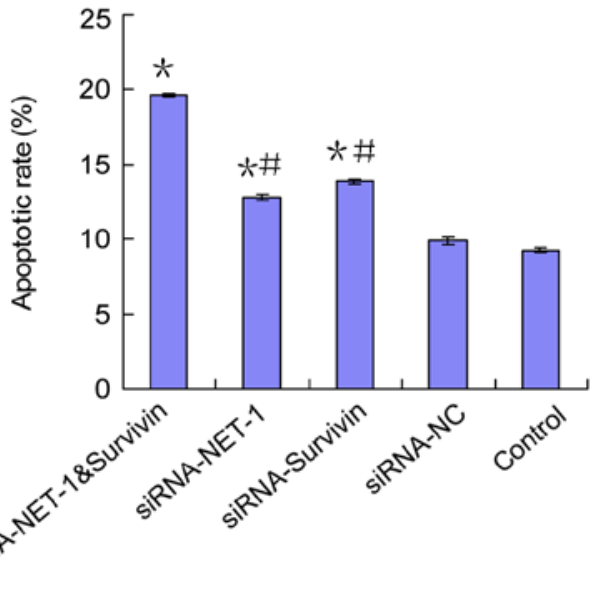

Figure 8. The apoptosis in A431 cells transfected with siRNA at $48 \mathrm{~h}$ by FCM. (A) FCM detected apoptosis of each group. (B) Histogram of A431 cell apoptosis ("p $<0.05$ vs. control; ${ }^{*} \mathrm{p}<0.05$ vs. siRNA-NET-1\&survivin). FCM, flow cytometry.

the effect of NET-1 siRNA, survivin siRNA and one-chaindouble-target siRNA on inducing A431 cell apoptosis. As shown in Fig. 8, treatment with NET-1 siRNA resulted in a significant increase by 1.39 -fold of apoptosis compared with that of the untreated cells $(\mathrm{p}<0.05)$. Similarly, an increase in apoptotic cells was identified after the survivin siRNA transfection compared to the untreated group. Furthermore, one-chaindouble-target siRNA-treated cells showed a higher increase of apoptosis, by 2.12-fold, compared to the untreated cells $(\mathrm{p}<0.05)$. There were significant differences in the apoptotic rates between one-chain-double-target siRNA-treated and NET-1 siRNA, or survivin siRNA-treated cells (all $\mathrm{p}<0.05$ ). No statistically significant difference was observed in A431 cell apoptosis between the siRNA-NC and blank control groups.

Detection of the protein expression by immunofluorescence staining and immunohistochemical staining in each group. As shown in Figs. 9 and 10, the expression of NET-1 and survivin was increased in SSCC cells in the untreated group, whereas the location of NET-1 and survivin varied from the membrane to cytoplasm, or mixed. These results were consistent with those of the gene microarray. A lower expression was observed in the NET-1 shRNA, survivin shRNA and dual-shRNAs groups as compared to that in the siRNA-NC and untreated groups. The one-chain-double-target siRNA therefore showed a higher inhibition of NET-1 and survivin expression as compared to that of NET-1 and survivin siRNA (all p<0.05). The difference between the expression of siRNA-NC and blank control groups was not statistically significant $(\mathrm{p}>0.05)$. The results showed that the immunofluorescence and immunohistochemical stainings were identical in the experiment.

\section{Discussion}

As a new member of the tetraspanins group, NET-1 is a recently identified tumor-associated gene. NET-1 is closely associated with the development and prognosis of several malignant tumors $(7-10,16)$. The overexpression of NET-1 in SSCC is involved in cancer cell proliferation (11).

Survivin is one of the most cancer-specific proteins identified thus far, being upregulated in almost all human tumors. Biologically, survivin has been shown to inhibit apoptosis, enhance proliferation and promote angiogenesis (13). Due to its upregulation in malignancy and its key role in apoptosis, proliferation and angiogenesis, survivin has been attracting considerable attention as a new target for anticancer therapies (17). 
A
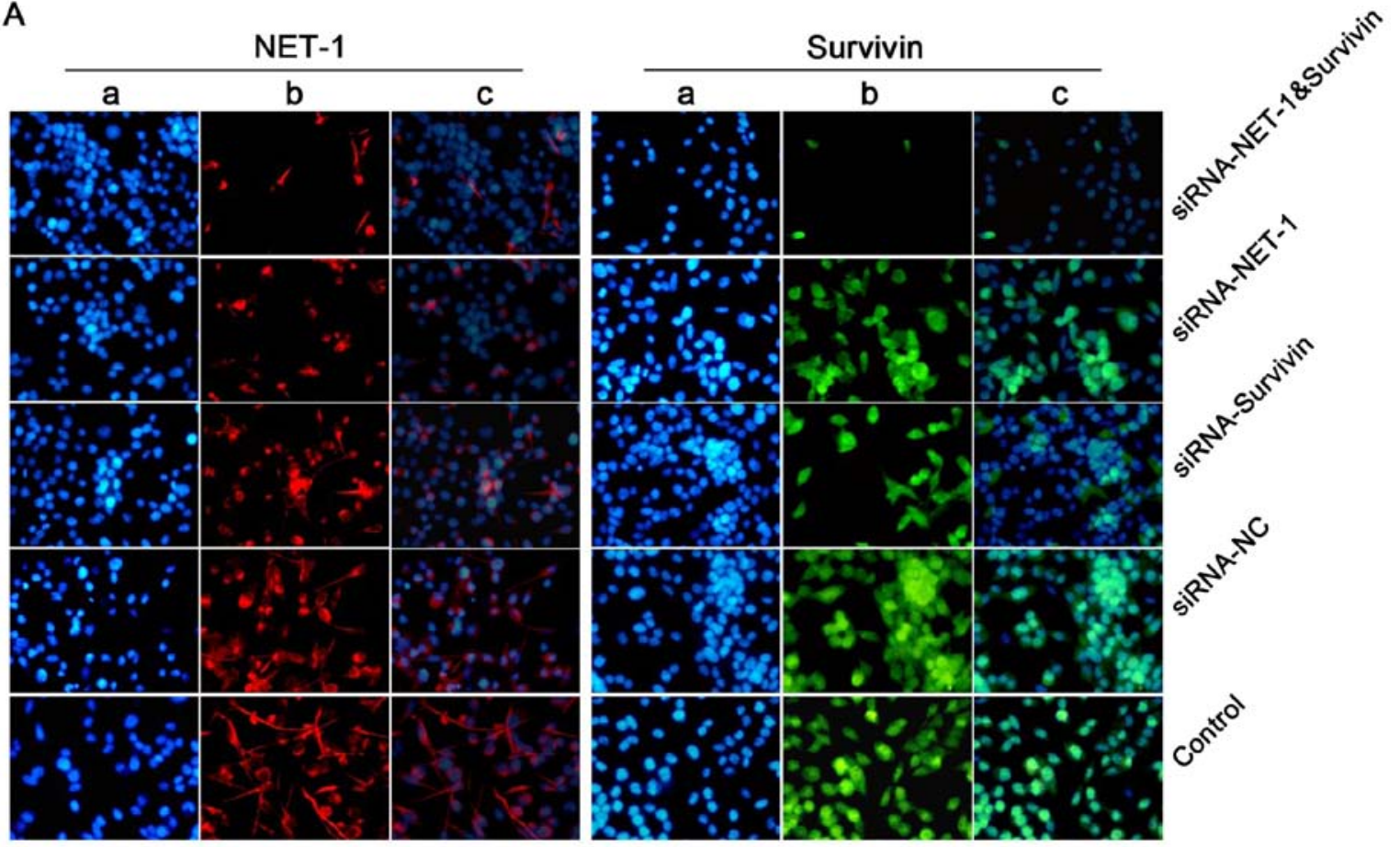

B

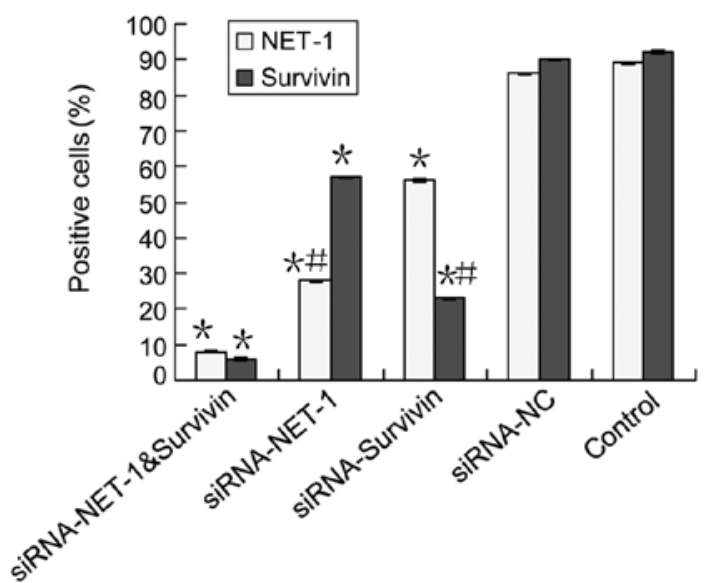

Figure 9. The protein localization and expression of NET-1 and survivin in A431 cells transfected with siRNA at 48 h. (A) Immunofluorescence staining, laser scanning confocal microscope (magnification, $x 400$ ): (a) nuclei are stained blue with Hoechst 33258, (b) NET-1 is marked red by TRITC located in the A431 cytoplasm and/or cell membrane, while survivin is marked green by FITC located in the A431 cytoplasm and/or cell membrane, (c) a is overlaid with b. (B) Histogram of positive cell percentage in A431 cells ( $\mathrm{p}<0.05$ vs. control; ${ }^{\#} \mathrm{p}<0.05$ vs. siRNA-NET-1\&survivin).

RNA interference (RNAi) is a sequence-specific posttranscriptional gene silencing mechanism that is triggered by double-stranded RNA (dsRNA) and causes degradation of homologous mRNA in sequence to the dsRNA. RNAi has emerged as one of the most important findings in the field of molecular biology (18). Due to its high efficacy and specificity in downregulating gene expression, RNAi was considered to be a potential therapeutic strategy against human cancer. Various individual oncogenes have been targeted by RNAi technology in different tumor cell models, leading to successful silencing of the protein and subsequently, cancer impairment. However, a number of these recent studies have selected a single gene rather than multiple genes as their targets. It is now generally accepted that there are many genes that are abnormally expressed in malignant tumors. In most cancers, silencing a single gene may be insufficient to therapeutically treat cancer cells. Therefore, simultaneously blocking multiple genes that are abnormally expressed may be more effective in the treatment of cancer cells than silencing a single gene. Previous findings have shown that simultaneously blocking multiple genes has a high protein inhibition as compared to silencing a single gene in cancer (19). Therefore, the application of vector-based RNAi technology involving multiple targets may be a promising therapeutic modality in the gene therapy of various types of cancer.

Due to the importance of the proliferation of NET-1 gene and anti-apoptotic survivin gene in tumor formation, the expression of cutaneous squamous cell carcinoma tissue chip was detected in 100 cases and a high expression of NET-1 and survivin was identified. Furthermore, this expression was 

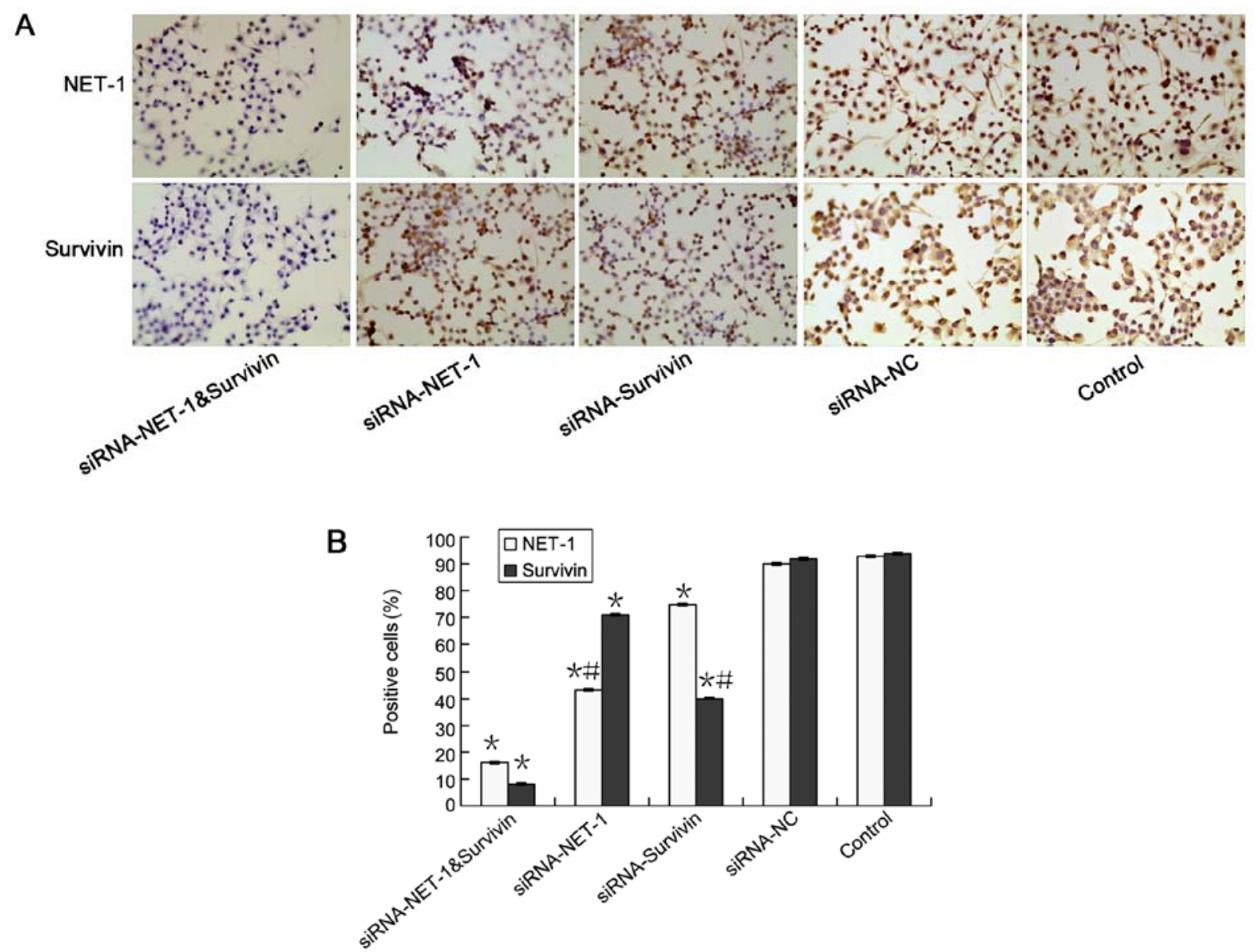

Figure 10. The protein localization and expression of NET-1 and survivin in A431 cells transfected with siRNA at 48 h. (A) Immunohistochemical staining (magnification, x400): nuclei are stained blue with hematoxylin; NET-1 and survivin are stained brown with DAD located in A431 cytoplasm and/or cell membrane. (B) Histogram of positive cell percentage in A431 cells ( ${ }^{*} \mathrm{p}<0.05$ vs. control; ${ }^{*} \mathrm{p}<0.05$ vs. siRNA-NET-1\&survivin).

positively correlated. This result indicated that the expression of NET-1 and survivin was closely associated with the degree of malignancy and biological behavior of cutaneous squamous cell carcinoma, indicating that the imbalance of the co-expression of NET-1 and survivin may be one of the mechanisms involved in the occurrence and development of SSCC. Previous results have shown that: the main role of NET-1 is to promote the proliferation of tumor cells, downregulate NET-1 gene expression and inhibit cell proliferation. By contrast, survivin has a strong anti-apoptotic role, downregulates survivin gene expression, and functions to promote cell apoptosis (pro-apoptotic and antiproliferative). Thus, a set of one-chain-double-target siRNAs was constructed aiming at the NET-1 and survivin genes in order to screen for effective single-target NET-1 siRNA and survivin siRNA sequences. The gene silencing effect of one-chain-double-target siRNA in cutaneous squamous cell carcinoma and its effect on tumor proliferation and apoptosis were assessed in in vitro studies.

In the present study, the RT-qPCR, western blotting, immunohistochemical and immunofluorescent assays showed that one-chain-double-target siRNA significantly inhibited the expression of NET-1, survivin mRNA and protein and the inhibitory effect was better than that of the single-target siRNA group, suggesting that one-chain-double-target siRNA was capable of closing two target genes simultaneously, and thus downregulating the expression of mRNA and protein more effectively. CCK- 8 and flow cytometry showed that after transfection with one-chain-double-target siRNA, the apoptotic rate of A431 cells was markedly increased and cell proliferation was significantly inhibited. The effects were better than those of the single target group, and the difference was of statistical significance. In addition, the RT-qPCR results showed that when NET-1 and survivin gene expression was inhibited, the expression of the angiogenic gene VEGF, proliferation gene cortactin and anti-apoptotic gene Bcl-2 were all decreased, while the expression of apoptotic genes caspase- 3 and -8 were increased.

VEGF is the most potent pro-angiogenic signal and was identified as a key angiogenic stimulator in cancer. Therefore, as VEGF is an effective target for SSCC therapy, a new approach to inhibit VEGF expression has to be developed (20). SSCC is a vascular tumor, with high invasion and metastasis, and the significant increase of angiogenesis in cancers may be closely associated with the malignant biological behavior of the tumor. Therefore, in the present study, the RT-qPCR result showed that VEGF levels of the double-target siRNA group were obviously decreased.

Cortactin has been described as an actin-associated scaffolding protein. It binds and activates the Arp2/3 complex and regulates the branched actin networks in the formation 
of dynamic cortical actin-associated structures (21). Cortactin participates in the regulation of actin cytoskeleton formation, and thereby the mechanism for controlling tumor cell migration, invasion and metastasis. The cortactin gene is the critical gene in the amplified chromosome 11q13 region responsible for increasing carcinoma motility and invasion (22). Cortactin excess expression may be associated with the deterioration of the tumor. Therefore, in the present study, the RT-qPCR result showed that cortactin levels of the double-target siRNA group were obviously decreased.

$\mathrm{Bcl}-2$ is a prominent member of a protein family that is responsible for the dysregulation of apoptosis and prevention of death in cancer cells (23). It controls the pathways leading to the release of cytochrome $c$ from the mitochondrial membrane, the activation of caspase cascade and eventually the execution of apoptosis. The flow cytometry results showed the percentage of apoptotic SSCC was significantly increased in all the treated groups compared with the untreated group. RT-qPCR showed the expression of caspase- 3 and -8 mRNA was significantly increased in the double-target group compared with the untreated group, which was consistent with the flow cytometric results, suggesting that NET-1 inhibits cell apoptosis through inactivation of the cell membrane receptor. NET-1 interference may lead to multimerization after Fas binding with FasL on SSCC surface and then bind with the cytoplasmic death domain binding protein, activate caspase- 8 and -3 , and induce SSCC apoptosis (24). Notably, the RT-qPCR results showed that the expression of Bcl-2 mRNA was markedly decreased in the double-target group, suggesting that NET-1 also inhibits cell apoptosis through the deactivation of mitochondria. NET-1 deficiency may cause the release of cytochrome $c$ from SSCC, which stimulates caspase-3 and -9 , leading to protein hydrolysis, DNA shear and activation of the intrinsic apoptosis pathway (25). Therefore, NET-1 inhibited the intrinsic and extrinsic apoptotic pathways of SSCC.

The above results suggest that there was a common signaling pathway between the NET-1 and survivin gene and the signals played a key role in processes such as tumor angiogenesis, cell proliferation, and apoptosis. Therefore, the downregulation or inhibition of the expression of NET-1 and survivin became an important strategy for the treatment of malignant tumors. On the other hand, NET-1 and survivin belong to the genes responsible for regulating cell proliferation and apoptosis. The immunohistochemistry results confirmed the existence of a correlation between NET-1 and survivin, and the co-immunoprecipitation results confirmed the existence of an interaction between NET-1 and survivin, suggesting that there was a common signaling pathway between the two genes and they were effective when combined. The combination can be synergized to improve the antitumor effect. Therefore, NET-1 and survivin may be combined as diagnostic and treatment indices for patients suffering from cutaneous squamous cell carcinoma.

In the present study, A431 cells were transfected with onechain-double-target siRNA, NET-1 single-target siRNA and survivin single-target siRNA. The NC siRNA constructed by Biomics Biotechnology Co., Ltd was used as the empty vector control group to exclude the effect of vector transfection on cell viability. The untransfected cells were used as a vehicle control group to exclude the effect of reagents on cell viability. The hypothesis of the present study was based on the understanding that excessive cell proliferation and apoptosis inhibition were key to tumor formation of tumors, and therefore the onechain-double-target siRNA designed for the proliferation of NET-1 and anti-apoptotic survivin genes may be important. The one-chain-double-target siRNA sequence had the properties of specificity, uniqueness and innovation and is conducive to the formation of therapeutic siRNA targeting cutaneous squamous cell carcinoma. One-chain-double-target siRNA targeting NET-1 and survivin genes expanded the RNAi technology, providing a foundation and experimental basis for the development of one-chain-multi-target siRNA technology.

In conclusion, the one-chain-double-target siRNA targeting NET-1 and survivin genes constructed in the present study was highly specific. The effect of double silencing of NET-1 and survivin genes was significantly enhanced compared with the single-target siRNA. The results may provide insight into the mechanisms and clinical treatment of cutaneous squamous cell carcinoma and other tumors that may be applied in further investigations. Future studies are required to create a model of nude mice bearing human squamous cell carcinoma, and examine the silencing effect of target genes caused by one-chain-double-targets siRNA in vivo by transdermal peptide transmission pathway. The ability of the tumor cells treated with one-chain-double-targets siRNA to resist chemotherapeutic drugs by ATP-TCA tumor susceptibility testing should also be investigated. We examined the manner in which the instability and in vivo complexity of one-chain-doubletargets siRNA may be overcome, thereby delivering siRNA accurately and safely to the target cells in order for RNAi to take effect in the local skin, which may contribute to prevention of problems in the circulatory system, be beneficial in the reduction of the administration dose and promote the development of one-chain-double-target siRNA technology. These methods may result in new treatment for patients suffering from skin cancer and other tumors.

\section{Acknowledgements}

The present study was supported by the foundation of the production-study-research prospective joint research programs of Jiangsu Province, China (BY 2013042-06).

\section{References}

1. Smoller BR: Squamous cell carcinoma: From precursor lesions to high-risk variants. Mod Pathol 19 (Suppl 2): S88-S92, 2006.

2. Bowen GM, White GL Jr and Gerwels JW: Mohs micrographic surgery. Am Fam Physician 72: 845-848, 2005.

3. Serru V, Dessen P, Boucheix C and Rubinstein E: Sequence and expression of seven new tetraspans. Biochim Biophys Acta 1478: 159-163, 2000.

4. Maecker HT, Todd SC and Levy S: The tetraspanin superfamily: Molecular facilitators. FASEB J 11: 428-442, 1997.

5. Yauch RL and Hemler ME: Specific interactions among transmembrane 4 superfamily (TM4SF) proteins and phosphoinositide 4-kinase. Biochem J 351: 629-637, 2000.

6. Chen L, Wang JL, Li H, Qin J and Wu YY: Functions of NET-1 gene in skin squamous cell carcinoma cell line (A431): A siRNA study. Zhonghua Bing Li Xue Za Zhi 38: 691-696, 2009 (In Chinese).

7. Chen L, Li X, Wang GL, Wang Y, Zhu YY and Zhu J: Clinicopathological significance of overexpression of TSPAN1, Ki67 and CD34 in gastric carcinoma. Tumori 94: 531-538, 2008 . 
8. Wollscheid V, Kühne-Heid R, Stein I, Jansen L, Köllner S, Schneider A and Dürst M: Identification of a new proliferationassociated protein NET-1/C4.8 characteristic for a subset of high-grade cervical intraepithelial neoplasia and cervical carcinomas. Int J Cancer 99: 771-775, 2002.

9. Chen L, Zhu YY, Zhang XJ, Wang GL, Li XY, He S, Zhang JB and Zhu JW: TSPAN1 protein expression: A significant prognostic indicator for patients with colorectal adenocarcinoma. World J Gastroenterol 15: 2270-2276, 2009.

10. Scholz CJ, Kurzeder C, Koretz K, Windisch J, Kreienberg R, Sauer G and Deissler H: Tspan-1 is a tetraspanin preferentially expressed by mucinous and endometrioid subtypes of human ovarian carcinomas. Cancer Lett 275: 198-203, 2009.

11. Chen L, Zhu Y, Li H, Wang GL, Wu YY, Lu YX, Qin J, Tuo J, Wang JL and Zhu J: Knockdown of TSPAN1 by RNA silencing and antisense technique inhibits proliferation and infiltration of human skin squamous carcinoma cells. Tumori 96: 289-295, 2010.

12. Kim GY, Lim SJ and Kim YW: Expression of HuR, COX-2, and survivin in lung cancers; cytoplasmic HuR stabilizes cyclooxygenase-2 in squamous cell carcinomas. Mod Pathol 24: 1336-1347, 2011.

13. Ryan BM, O'Donovan N and Duffy MJ: Survivin: A new target for anti-cancer therapy. Cancer Treat Rev 35: 553-562, 2009.

14. Cheng K and Mahato RI: Gene modulation for treating liver fibrosis. Crit Rev Ther Drug Carrier Syst 24: 93-146, 2007.

15. Mahato RI, Cheng K and Guntaka RV: Modulation of gene expression by antisense and antigene oligodeoxynucleotides and small interfering RNA. Expert Opin Drug Deliv 2: 3-28, 2005.

16. Chen L, Wang Z, Zhan X, Li DC, Zhu YY and Zhu J: Association of NET-1 gene expression with human hepatocellular carcinoma. Int J Surg Pathol 15: 346-353, 2007.
17. Zaffaroni N, Pennati M and Daidone MG: Survivin as a target for new anticancer interventions. J Cell Mol Med 9: 360-372, 2005.

18. Wu JB, Fu HQ, Huang LZ, Liu AW and Zhang JX: Effects of siRNA-targeting BMP-2 on the abilities of migration and invasion of human liver cancer SMMC7721 cells and its mechanism. Cancer Gene Ther 18: 20-25, 2011.

19. Chen SM, Wang Y, Xiao BK and Tao ZZ: Effect of blocking VEGF, hTERT and Bcl-xl by multiple shRNA expression vectors on the human laryngeal squamous carcinoma xenograft in nude mice. Cancer Biol Ther 7: 734-739, 2008.

20. Takahashi $\mathrm{H}$ and Shibuya M: The vascular endothelial growth factor (VEGF)/VEGF receptor system and its role under physiological and pathological conditions. Clin Sci 109: 227-241, 2005.

21. Yamada S, Yanamoto S, Kawasaki G, Mizuno A and Nemoto TK Overexpression of cortactin increases invasion potential in oral squamous cell carcinoma. Pathol Oncol Res 16: 523-531, 2010.

22. Hsu KF, Lin CK, Yu CP, Tzao C, Lee SC, Lee YY, Tsai WC and Jin JS: Cortactin, fascin, and survivin expression associated with clinicopathological parameters in esophageal squamous cell carcinoma. Dis Esophagus 22: 402-408, 2009.

23. Thomas S, Quinn BA, Das SK, Dash R, Emdad L, Dasgupta S, Wang XY, Dent P, Reed JC, Pellecchia M, et al: Targeting the Bcl-2 family for cancer therapy. Expert Opin Ther Targets 17: $61-75,2013$

24. Ghavami S, Hashemi M, Ande SR, Yeganeh B, Xiao W, Eshraghi M, Bus CJ, Kadkhoda K, Wiechec E, Halayko AJ, et al: Apoptosis and cancer: Mutations within caspase genes. J Med Genet 46: 497-510, 2009.

25. Jeong SY and Seol DW: The role of mitochondria in apoptosis. BMB Rep 41: 11-22, 2008. 